\title{
Dynamical analysis of homogenized second gradient anisotropic media for textile composite structures and analysis of size effects
}

\author{
H. Reda ${ }^{\mathrm{a}, \mathrm{b}}$, I. Goda ${ }^{\mathrm{c}, \mathrm{d}}$, J.F. Ganghoffer ${ }^{\mathrm{a}, *}$, G. L'Hostis $^{c}$, H. Lakiss $^{\mathrm{b}}$ \\ ${ }^{a}$ LEMTA, Université de Lorraine, 2, Avenue de la Forêt de Haye. TSA 60604, 54504 Vandoeuvre-les-Nancy, France \\ ${ }^{\mathrm{b}}$ Faculty of Engineering, Section III, Lebanese University, Campus Rafic Hariri, Beirut, Lebanon \\ ${ }^{\mathrm{c}}$ LPMT, Université de Haute-Alsace, 11 rue Alfred Werner, 68093 Mulhouse Cedex, France \\ ${ }^{\mathrm{d}}$ Department of Industrial Engineering, Faculty of Engineering, Fayoum University, Fayoum 63514, Egypt
}

In order to predict the dispersion relation of 3D composite structures in the low frequency range, we con-struct effective first and second order grade continuum models. The effective properties of textile com-posites are obtained computationally by an equivalent strain energy method based on the response of the representative volume unit cell (RUC) under prescribed boundary conditions as described in Goda and Ganghoffer (2016). The expressions of the phase velocities for the three modes of wave propagation in a 3D context (longitudinal, horizontal shear and vertical shear) reveal that the second order continuum is dispersive, due to the presence of the second order elasticity constants. The shape change of the phase velocity when increasing the wave number shows the dispersive behavior of the second gradient med-ium, whereas Cauchy medium is non dispersive. Plots of the iso-frequency contour for the two investi-gated composites in the case of second gradient and Cauchy effective medium show that the second gradient contributions does not modify the anisotropic behavior of the considered composites. Important size effects on the dynamical behavior are shown, especially reflected by the dispersive behav-ior and the anisotropic dynamic responses, due to the significant overall increase of the second order rigidity matrix when increasing the RUC size.

\section{Introduction}

Architectured materials, and specially periodic network materials used as acoustics metamaterials are widely used in engineering applications, like vibration control devices or energy absorption systems [1,2], for noise reduction purpose [3,4]. They also find some use to control and manipulate the propagation of elastic waves into different kind of structures [5-7]. One of their interesting properties is the appearance of band gaps: within some frequency interval, waves cannot propagate through the network. A lot of attention has been paid to study the static and dynamic behavior of $2 \mathrm{D}$ periodic structures, but the investigation of $3 \mathrm{D}$ structures for the control of waves is one of the emerging topics in acoustics metamaterials, especially since difficulties occur when resorting to the wave finite element method [8-12], due to the huge induced numerical cost. This is the main motivation to model the dynamical behavior of such 3D complex structures using the effective continuum medium, in situations when the wave length is greater than the network unit cell size.

\footnotetext{
* Corresponding author.

E-mail address: jean-francois.ganghoffer@univ-lorraine.fr (J.F. Ganghoffer).
}

In the low frequency range, in order to predict the dispersion relation of the effective continuum enriched by second gradient elasticity to account for microstructural effects, the construction of the first and second effective rigidity matrix is needed. Different homogenization methods have been proposed to build continuum description of periodic discrete materials [13-15]. We use in this contribution an energetic method based on the computation of the strain energy of the unit cell to predict the components of the first and second rigidity matrix components [16] of the constitutive law of the effective continuum model enriched by the second gradient displacement. Key feature of this non-standard theory is the introduction of higher order gradients of the displacement field, accompanied by internal length scales [17,18]. A large amount of recent works in the literature devoted to gradient elasticity applied to dynamics can be found e.g. in [19-22].

Wave propagation analysis has been limited so far in the literature to 2D periodic structures [23,24], which are replaced by a classical Cauchy-type effective elastic medium, involving the sole first order displacement gradient. Models based on Cauchy-type theory do however not give realistic predictions of the medium properties such as wave dispersion. It has been shown that generalized continuum theories, that constitute an extension of the 


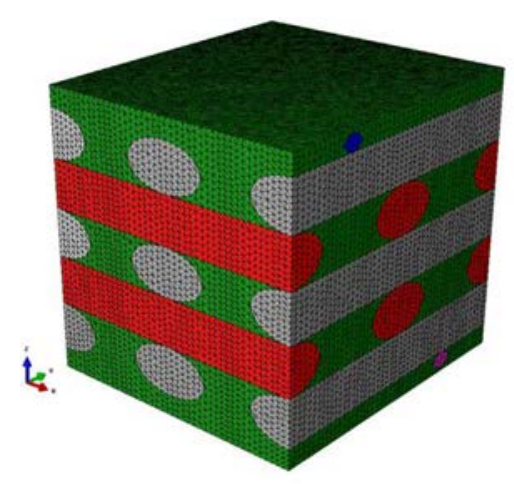

(a)

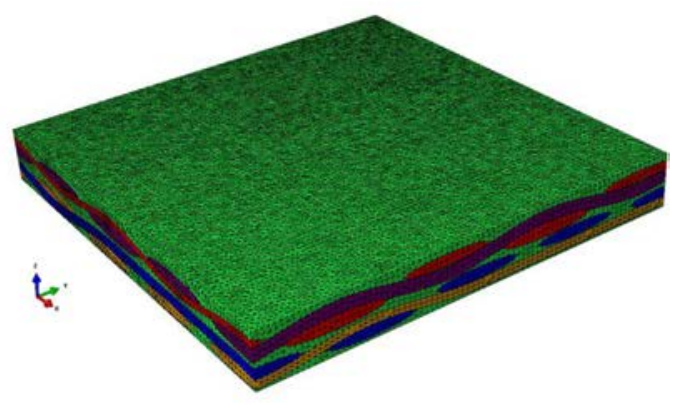

(b)

Fig. 1. (a) Woven 3D interlocks, and (b) Layered textile composite for which first and second-gradient elastic moduli are identified.

classical continuum of elasticity by including additional degrees of freedom or additional higher-order gradients of the displacements, offer an attractive alternative for capturing size effects, strain and stress fields in the neighborhood of singularities [25-28] and wave dispersion in a dynamic context [29-34], these effects being overlooked by classical elasticity. The Cauchy medium is indeed nondispersive, which means that waves propagate independently of the frequency of the incident wave [35]. It has however been proven by experiments that all waves are dispersive, that is, each frequency travels with a different phase velocity [36,37]. This explains the success of gradient-enriched theories in capturing dynamic behaviors overlooked by classical elasticity.

The present work is organized as follows: we expose in Section 2 an energetic method based on the strain energy of the microstructured solids to compute the homogenized first and second order rigidity tensors. We next introduce the computed effective constitutive laws into the dynamical planar equilibrium equations, and formulate the wave motion equation reflecting the propagation of three modes of waves within the constructed effective medium. We next (Section 3) an application on the layered textile composite and the 3D interlock Fig. 1 is made and the analysis of free wave motion at low frequencies is done in terms of the dispersion relation. The comparison between the Cauchy medium and the second gradient in terms of the dispersive and anisotropic behavior of the considered microstructures is done in Section 4. The influence of the size of the unit cell on the effective mechanical properties and dispersion relation are analyzed in Section 5. We conclude in Section 6 by a summary of the main results and a few perspectives for future developments.

\section{Basic equations of strain-gradient elasticity}

In this section, we recall the basic equations of strain-gradient elasticity, including the constitutive relations; these relations involve the classical fourth-order elasticity tensor supplemented by a fifth-order coupling tensor and a sixth-order tensor relating the hyperstress tensor to the strain gradient tensor.

\subsection{Constitutive relations and internal lengths}

In classical elasticity theory which involves the sole firstgradient of displacement, stress at a material point is linked to strain through the classical elasticity tensor. The second-grade elasticity is a kinematic enhancement of classical elasticity taking into account the second gradient of displacement in the mechanical formulation. In the strain-gradient theory of linear elasticity, the constitutive law provides the symmetric Cauchy stress tensor $\boldsymbol{\sigma}$ and the hyper-stress (or double stress) tensor $\boldsymbol{\sigma}^{s}$. They are next given in terms of the infinitesimal strain tensor $\boldsymbol{\varepsilon}$ and straingradient tensor $\nabla \varepsilon$.

Tensors $\boldsymbol{\sigma}$ and $\boldsymbol{\sigma}^{s}$ are related to $\boldsymbol{\varepsilon}$ and $\boldsymbol{K}=\boldsymbol{\nabla} \boldsymbol{\varepsilon}$ through the following general constitutive law for a homogeneous anisotropic second order grade continuum:

$\sigma_{i j}=C_{i j l m} \varepsilon_{l m}+M_{i j l m n} K_{l m n}$
$\sigma_{i j k}^{s}=M_{i j k l m} \varepsilon_{l m}+D_{i j k l m n} K_{l m n}$

with $C_{i j l m}$ the classical fourth-order elastic tensor, $D_{i j k l m n}$ the sixthorder second order elastic tensor, and $M_{i j l m n}$ the fifth-order coupling tensor between first and second order elasticity behaviors.

The aforementioned terms in (1) $\sigma_{i j}, \varepsilon_{i j}, \sigma_{i j k}^{s}$ and $K_{i j k}=\varepsilon_{i j, k}$ are respectively, the components of the corresponding tensors $\boldsymbol{\sigma}, \boldsymbol{\varepsilon}$, $\boldsymbol{\sigma}^{s}$ and $\boldsymbol{K}=\nabla \boldsymbol{\varepsilon}$ relative to a selected orthonormal basis $\left\{\mathbf{e}_{1}, \mathbf{e}_{2}, \mathbf{e}_{3}\right\}$ of a 3-dimensional Euclidean space. The matrix components $C_{i j l m}, M_{i j l m n}, D_{i j k l m n}$ further have the following index permutation symmetry properties:

$C_{i j l m}=C_{j i l m}=C_{l m i j}$;

$M_{i j k l m}=M_{j i k l m}=M_{i j l k m}$;

$D_{i j k l m n}=D_{j i k l m n}=D_{\text {lmnijk }}$

In the case where the microstructure of a material exhibits central symmetry, the fifth-order coupling elastic stiffness tensor $\mathbf{M}$ vanishes, so that the previous constitutive law becomes uncoupled

$\sigma_{i j}=C_{i j l m} \varepsilon_{l m}$

$\sigma_{i j k}^{s}=D_{i j k l m n} K_{l m n}$

Next, the strain gradient theory is exploited in terms of the strain and second gradient of the displacement field which are, respectively, the second and third order tensors

$$
\begin{aligned}
& \boldsymbol{\varepsilon}=\frac{1}{2}(\boldsymbol{u} \otimes \nabla+\boldsymbol{u} \otimes \nabla), \quad \boldsymbol{K}=\boldsymbol{u} \otimes \nabla \otimes \nabla \\
& \Longleftrightarrow \varepsilon_{i j}=\frac{1}{2}\left(u_{i, j}+u_{j, i}\right), \quad K_{i j k}=\varepsilon_{i j, k}=\frac{1}{2}\left(u_{i, j k}+u_{j, i k}\right)
\end{aligned}
$$

The classical infinitesimal strain tensor $\boldsymbol{\varepsilon}$ is the symmetric part of the displacement field gradient and it has six independent components in a three dimensional situation. The second gradient of the displacement field, tensor $\boldsymbol{K}$, is symmetric in the last two indices and has eighteen independent components, so that it holds the symmetries $\varepsilon_{i j}=\varepsilon_{j i}, K_{i j k}=K_{i k j}$.

In three dimensions (3D), the displacement field is the vector $\boldsymbol{u}=\left[\begin{array}{lll}u_{x} & u_{y} & u_{z}\end{array}\right]^{T}$, which entails the following strain tensor in vector form

$\boldsymbol{\varepsilon}=\left[\varepsilon_{x x}, \varepsilon_{y y}, \varepsilon_{z z}, \varepsilon_{x y}=\varepsilon_{y x}, \varepsilon_{y z}=\varepsilon_{z y}, \varepsilon_{x z}=\varepsilon_{z x}\right]^{T}$ 
The strain tensor is symmetrical with components depending upon the displacements gradients; it is written from the first gradient of displacement in vector form as

$\boldsymbol{\varepsilon}=\left[\frac{\partial u_{x}}{\partial x}, \frac{\partial u_{y}}{\partial y}, \frac{\partial u_{z}}{\partial z}, \frac{1}{2}\left(\frac{\partial u_{y}}{\partial x}+\frac{\partial u_{x}}{\partial y}\right), \frac{1}{2}\left(\frac{\partial u_{z}}{\partial y}+\frac{\partial u_{y}}{\partial z}\right), \frac{1}{2}\left(\frac{\partial u_{z}}{\partial x}+\frac{\partial u_{x}}{\partial z}\right)\right]^{T}$

In the same manner, the strain gradient tensor is written in vector format from the second gradient of the displacement field as

$\boldsymbol{K}=\left[\frac{\partial^{2} u_{x}}{\partial x^{2}}, \frac{\partial^{2} u_{y}}{\partial y^{2}}, \frac{\partial^{2} u_{z}}{\partial z^{2}}, \frac{\partial^{2} u_{x}}{\partial y^{2}}, \frac{\partial^{2} u_{x}}{\partial z^{2}}, \frac{\partial^{2} u_{y}}{\partial x^{2}}, \frac{\partial^{2} u_{y}}{\partial z^{2}}, \frac{\partial^{2} u_{z}}{\partial x^{2}}, \frac{\partial^{2} u_{z}}{\partial y^{2}}, \frac{\partial^{2} u_{x}}{\partial x \partial y}\right.$,

$$
\left.\frac{\partial^{2} u_{x}}{\partial x \partial z}, \frac{\partial^{2} u_{x}}{\partial y \partial z}, \frac{\partial^{2} u_{y}}{\partial x \partial y}, \frac{\partial^{2} u_{y}}{\partial x \partial z}, \frac{\partial^{2} u_{y}}{\partial y \partial z}, \frac{\partial^{2} u_{z}}{\partial x \partial y}, \frac{\partial^{2} u_{z}}{\partial x \partial z}, \frac{\partial^{2} u_{z}}{\partial y \partial z}\right]^{T}
$$

The stress and hyper-stress for the effective 3D second order grade continuum can be defined in vector format as

$$
\begin{aligned}
\boldsymbol{\sigma}=[ & {\left[\sigma_{x x}, \sigma_{y y}, \sigma_{z z}, \sigma_{x y}, \sigma_{y z}, \sigma_{x z}\right]^{T}, } \\
\boldsymbol{\sigma}^{s}= & {\left[\sigma_{x x x}^{s}, \sigma_{y y y}^{s}, \sigma_{z z z}^{s}, \sigma_{x y y}^{s}, \sigma_{x z z}^{s}, \sigma_{y x x}^{s}, \sigma_{y z z}^{s}, \sigma_{z x x}^{s}, \sigma_{z y y}^{s}, \sigma_{x x y}^{s}, \sigma_{x x z}^{s}, \sigma_{x y z}^{s},\right.} \\
& \left.\sigma_{y x y}^{s}, \sigma_{y x z}^{s}, \sigma_{y y z}^{s}, \sigma_{z x y}^{s}, \sigma_{z x z}^{s}, \sigma_{z y z}^{s}\right]^{T}
\end{aligned}
$$

Regarding notations, the comma denotes the partial derivative.

The static equilibrium equations write in index format and in the absence of body forces

$\sigma_{i k, k}-\sigma_{i j k, j k}^{S}=0$

Inserting therein the constitutive law for the stress and hyperstress, leads to the equilibrium equations in terms of the spatial gradients of the strain tensor,

$C_{i k p q} \varepsilon_{p q, k}-D_{i j k p q r} K_{p q r . j k}=0$

Due to the kinematic relations $K_{i j k}=\varepsilon_{i j, k}=\frac{1}{2}\left(u_{i, j k}+u_{j, i k}\right)$, previous equilibrium equation (9) rewrites

$\left.C_{i k p q} \varepsilon_{p q, k}-D_{i j k p q r} \varepsilon_{p q, r j k}=0 \rightarrow C_{i k p q} 1-\frac{D_{i j k p q r}}{C_{i k p q}} \frac{\partial^{2}}{\partial x_{r} \partial x_{j}}\right) \varepsilon_{p q, k}=0$

It holds the following minor and major symmetries for the elasticity tensors:

$$
\begin{aligned}
& C_{j k p q}=C_{k j p q}=C_{j k q p}=C_{p q j k} \\
& D_{i j k p q r}=D_{\underline{(i j) k(p q) r}}=D_{\underline{\alpha k \beta r}}, \quad \alpha, \beta=1 \ldots 6, \quad k, r=1 \ldots 3
\end{aligned}
$$

introducing therein Voigt notation with the correspondence of indices $\alpha \leftrightarrow(i j), \beta \leftrightarrow(p q)$, the parenthesis therein denoting the symmeterization of the enclosed indices, and using the major symmetry of the $\mathbf{D}$ tensor of second order elastic moduli. The pair of indices $\alpha k, \beta r$ which are underlined can be exchanged, so that it holds the equality $D_{\alpha k \beta r}=D_{\beta r_{\alpha k}}$. This accordingly defines a tensor D represented as a $\overline{18} \times \overline{18}$ square matrix.

To summarize, the tensor of classical first order moduli can be represented as a symmetrical second order tensor with 21 independent coefficients, and the sixth order tensor of second order elastic moduli can be represented as a 18 by $18 \mathrm{~s}$ order symmetrical tensor, having accordingly 171 independent components for a fully anisotropy effective continuum. The number of truly independent components is then reduces by examining the material symmetries of the considered structure.

Due to previous considerations, the equilibrium equation takes the equivalent form
$\left.\left.C_{i k p q} \quad 1-\frac{D_{i j k p q r}}{C_{i k p q}} \frac{\partial^{2}}{\partial x_{r} \partial x_{j}}\right) \varepsilon_{p q, k}=0 \Longleftrightarrow C_{\alpha \beta} \quad 1-\frac{D_{\alpha k \beta r}}{C_{i k p q}} \frac{\partial^{2}}{\partial x_{r} \partial x_{j}}\right) \varepsilon_{\beta, k}=0$

introducing therein the Voigt notation with the Greek indices taking values from 1 to 6 , and latin indices from 1 to 3 ; the parenthesis denote the symmeterization of the enclosed indices, and we have used the previously mentioned major symmetry of the tensor of second order elastic moduli.

The six internal length associated to the independent classical moduli $C_{\alpha \beta}$ can then be identified by the relations

$\left.l_{\alpha \beta}=\sum_{r=1}^{3} D_{\alpha r \beta r} / C_{\alpha \beta}\right)^{1 / 2}$

The last equality follows from the major symmetry of the second order elasticity coefficients.

Introducing these internal lengths entails that the previous equilibrium equations rewrite

$C_{\alpha \beta}\left(1-l_{\alpha \beta}^{2} \nabla^{2}\right) \varepsilon_{\beta, j}=0$

with $\nabla$ therein the nabla operator. We note that the material length scale parameters appear as the ratio of second order to first order equivalent moduli; their direct identification from the computed equivalent tensors of first and second order gradient rigidities is however challenging for a general anisotropic medium, so that the present general methodology is useful for this purpose.

\subsection{Wave propagation in the second gradient effective medium}

In order to determine the dispersion relation and the group and phase velocities for propagating waves, we write the dynamical equilibrium equation for 3D structures, as predicted by second gradient continuum. Relying on the constitutive equations of the obtained continuum second gradient medium (2), the dynamical equations of motion write in components form as the three following differential equations along the $\mathrm{x}, \mathrm{y}$ and $\mathrm{z}$ directions of a Cartesian coordinates system,

$$
\begin{gathered}
\left(\frac{\partial \sigma_{x x}}{\partial x}+\frac{\partial \sigma_{x y}}{\partial y}+\frac{\partial \sigma_{x z}}{\partial z}\right)-\frac{\partial^{2} \sigma_{x x x}^{s}}{\partial x^{2}}-\frac{\partial^{2} \sigma_{x y y}^{s}}{\partial y^{2}}-\frac{\partial^{2} \sigma_{x z z}^{s}}{\partial z^{2}}-\frac{\partial^{2} \sigma_{x x y}^{s}}{\partial x \partial y} \\
-\frac{\partial^{2} \sigma_{x x z}^{s}}{\partial x \partial z}-\frac{\partial^{2} \sigma_{x y z}^{s}}{\partial y \partial z}=\rho^{*} \ddot{u}_{x} \\
\left(\frac{\partial \sigma_{y x}}{\partial x}+\frac{\partial \sigma_{y y}}{\partial y}+\frac{\partial \sigma_{y z}}{\partial z}\right)-\frac{\partial^{2} \sigma_{y y y}^{s}}{\partial y^{2}}-\frac{\partial^{2} \sigma_{y x x}^{s}}{\partial x^{2}}-\frac{\partial^{2} \sigma_{y z z}^{s}}{\partial z^{2}}-\frac{\partial^{2} \sigma_{y x y}^{s}}{\partial x \partial y} \\
-\frac{\partial^{2} \sigma_{y x z}^{s}}{\partial x \partial z}-\frac{\partial^{2} \sigma_{y y z}^{s}}{\partial y \partial z}=\rho^{*} \ddot{u}_{y} \\
\left(\frac{\partial \sigma_{z x}}{\partial x}+\frac{\partial \sigma_{z y}}{\partial y}+\frac{\partial \sigma_{z z}}{\partial z}\right)-\frac{\partial^{2} \sigma_{z z z}^{s}}{\partial z^{2}}-\frac{\partial^{2} \sigma_{z x x}^{s}}{\partial x^{2}}-\frac{\partial^{2} \sigma_{z y y}^{s}}{\partial y^{2}}-\frac{\partial^{2} \sigma_{z x y}^{s}}{\partial x \partial y} \\
-\frac{\partial^{2} \sigma_{z x z}^{s}}{\partial x \partial z}-\frac{\partial^{2} \sigma_{z y z}^{s}}{\partial y \partial z}=\rho^{*} \ddot{u}_{z}
\end{gathered}
$$

The second order time derivatives $\ddot{u}_{x}, \ddot{u}_{y}$ and $\ddot{u}_{z}$ therein are the components of the acceleration vector. The effective mass density $\rho^{*}$ therein of a composite is simply the volume average of its constituents densities.

For a harmonic plane wave propagating along an axis in an infinite planar second gradient medium, the displacement field with components $q=\left(u_{x}, u_{y}, u_{z}\right)$ at a point $\mathbf{r}$ assumed in the following form as

$q=\widehat{Q} e^{i(\omega t-\mathbf{k r})}$ 


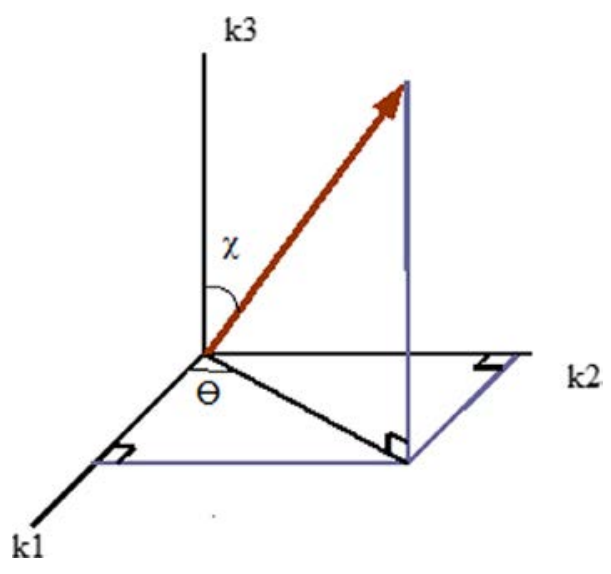

Fig. 2. Direction of propagation in 3D space defined by the two angular variables $\theta$ and $\chi$.

In (15), $\widehat{Q}=(\widehat{U}, \widehat{V}, \widehat{W})$ is the amplitude of the wave motion in the $\mathrm{x}, \mathrm{y}$ and $\mathrm{z}$ direction respectively, $\mathbf{k}=\left(k_{1}, k_{2}, k_{3}\right)$ the wave vector and $\omega$ the frequency. The wave vector is a complex number; its real part represents the phase constants and its imaginary parts represent the space attenuation in Cartesian coordinate system.

Substituting the plane harmonic wave (15) and the constitutive law (2) in the dynamical equations (14) leads to a wave motion equation describing the propagation of longitudinal, vertical and horizontal shear waves as:

$$
\left(\begin{array}{ccc}
A-\rho^{*} \omega^{2} & \frac{1}{2} \mathrm{k}_{1} \mathrm{k}_{2}\left(2 \mathrm{C}_{12}+\mathrm{C}_{44}\right) & \frac{1}{2} \mathrm{k}_{1} \mathrm{k}_{3}\left(2 \mathrm{C}_{13}+\mathrm{C}_{66}\right) \\
\frac{1}{2} \mathrm{k}_{1} \mathrm{k}_{2}\left(2 \mathrm{C}_{12}+\mathrm{C}_{44}\right) & B-\rho^{*} \omega^{2} & \frac{1}{2} \mathrm{k}_{2} \mathrm{k}_{3}\left(2 \mathrm{C}_{23}+\mathrm{C}_{55}\right) \\
\frac{1}{2} \mathrm{k}_{1} \mathrm{k}_{3}\left(2 \mathrm{C}_{13}+\mathrm{C}_{66}\right) & \frac{1}{2} \mathrm{k}_{2} \mathrm{k}_{3}\left(2 \mathrm{C}_{23}+\mathrm{C}_{55}\right) & C-\rho^{*} \omega^{2}
\end{array}\right)\left(\begin{array}{c}
\widehat{\mathrm{U}} \\
\widehat{\mathrm{V}} \\
\widehat{W}
\end{array}\right)=0
$$

with the coefficients therein given by the expressions

$$
\begin{aligned}
A= & C_{66} k_{1}^{2}+\frac{1}{2} C_{44} k_{2}^{2}+\frac{1}{2} C_{66} k_{3}^{2}+D_{111} k_{1}^{4}+D_{112} k_{1}^{2} k_{2}^{2}+D_{113} k_{1}^{2} k_{3}^{2} \\
& +D_{122} k_{2}^{4}+D_{123} k_{2}^{2} k_{3}^{2}+D_{133} k_{3}^{4} \\
B= & \frac{1}{2} C_{44} k_{1}^{2}+C_{22} k_{2}^{2}+\frac{1}{2} C_{55} k_{3}^{2}+D_{222} k_{2}^{4}+D_{211} k_{1}^{4}+D_{233} k_{3}^{4} \\
& +D_{212} k_{1}^{2} k_{2}^{2}+D_{213} k_{1}^{2} k_{3}^{2}+D_{223} k_{2}^{2} k_{3}^{2} \\
C= & \frac{1}{2} C_{66} k_{1}^{2}+\frac{1}{2} C_{55} k_{2}^{2}+C_{33} k_{3}^{2}+D_{322} k_{2}^{4}+D_{311} k_{1}^{4}+D_{333} k_{3}^{4} \\
& +D_{312} k_{1}^{2} k_{2}^{2}+D_{313} k_{1}^{2} k_{3}^{2}+D_{323} k_{2}^{2} k_{3}^{2}
\end{aligned}
$$

Nontrivial solutions of the system of Eq. (16) exist if and only if its determinant $\Delta\left(\omega, k_{1}, k_{2}, k_{3}\right)$ vanishes; the obtained positive roots characterize the dispersion relations for planar wave propagation. Three modes of propagation exist, namely the longitudinal mode (designated by L), the vertical shear mode (labeled SV), and the horizontal shear mode ( $\mathrm{SH}$ in short). The phase and group velocities are defined as follows

$$
\begin{aligned}
& c^{p}=\frac{w}{|k|}, \quad c^{g}=\left(\frac{\partial \omega}{\partial k_{1}}, \frac{\partial \omega}{\partial k_{2}}, \frac{\partial \omega}{\partial k_{3}}\right) \\
& |k|=\sqrt{k_{1}^{2}+k_{2}^{2}+k_{3}^{2}}
\end{aligned}
$$

The wave vector $\mathrm{k}$ is a complex number: its real part represents the attenuation in a Cartesian coordinate system in 3D, whereas its imaginary part represents the phase constants.

For a plane wave without attenuation in the 3D Cartesian coordinate system, the propagation constants along the $x, y, z$ directions are

$$
\begin{aligned}
& k_{1}=i \varepsilon_{1}=|k| \sin (\chi) \cos (\theta), \quad k_{2}=i \varepsilon_{2}=|k| \sin (\chi) \sin (\theta), \\
& k_{3}=i \varepsilon_{3}=|k| \cos (\chi)
\end{aligned}
$$

with the parameterization shown in Fig. 2.

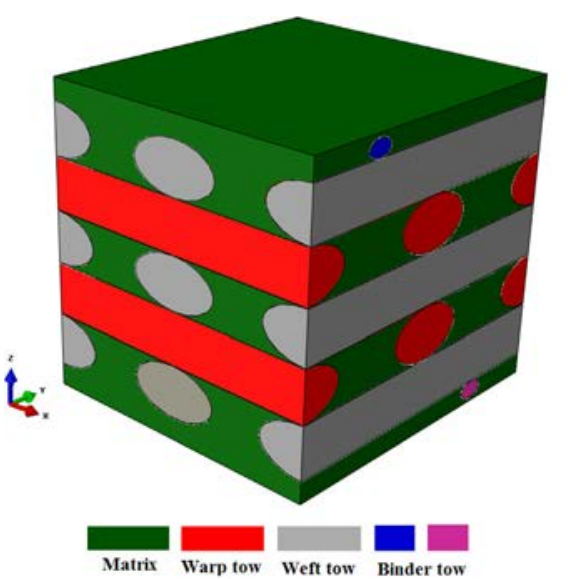

(a)

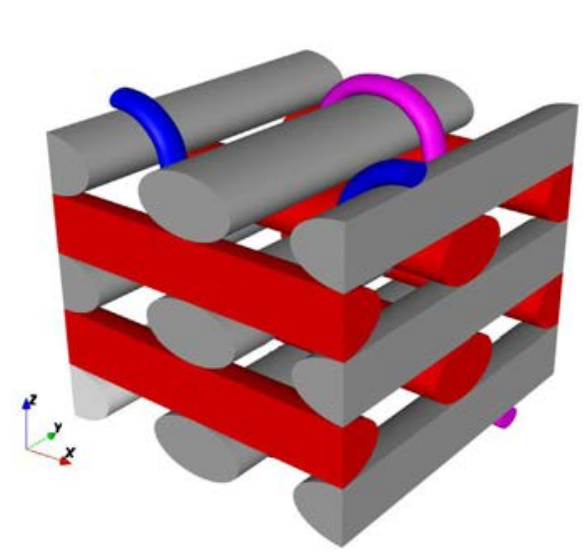

(b)

Fig. 3. (a) Elementary cell of the 3D orthogonal impregnated textile composed of dry textile and resin. (b) Dry textile architecture.

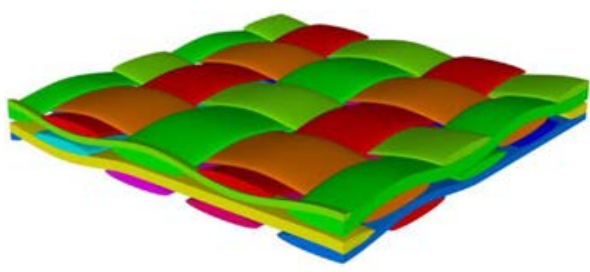

(a)

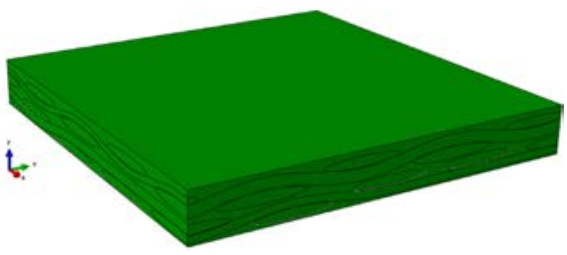

(b)

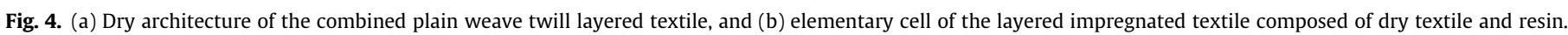


In the case of propagation in the longitudinal direction (the $\mathrm{x}$ direction in the Cartesian frame), the dispersive longitudinal mode (proportional to the wavenumber $\mathrm{k}$ ) is controlled by the components of the rigidity matrix in traction along $\mathrm{x}$, a mode with frequency $\omega_{l}=\sqrt{\frac{C_{11}+D_{111} k_{1}^{2}}{\rho^{*}}} k_{1}$, while the dispersive vertical shear mode is controlled by the shear components along the y direction, with frequency given by $\omega_{S V}=\frac{\sqrt{2}}{2} \sqrt{\frac{C_{44}+2 D_{211} k_{1}^{2}}{\rho^{*}}} k_{1}$; lastly, for the

Table 1

Constituent material properties of the RUC.

\begin{tabular}{llll}
\hline Constituent & Material & Young's modulus (MPa) & Poisson's ratio \\
\hline Matrix & Epoxy & 2600 & 0.4 \\
Inclusion & E-glass & 72,500 & 0.22 \\
\hline
\end{tabular}

Table 2

Geometrical parameters of the 3D through-thickness orthogonal interlock composite unit cell.

\begin{tabular}{llll}
\hline & $\begin{array}{l}\text { Width } \\
{[\mathrm{mm}]}\end{array}$ & $\begin{array}{l}\text { Thickness } \\
{[\mathrm{mm}]}\end{array}$ & $\begin{array}{l}\text { Spacing } \\
{[\mathrm{mm}]}\end{array}$ \\
\hline Warp & 0.25 & 0.15 & 0.473 \\
Weft & 0.27 & 0.15 & 0.429 \\
Binder & 0.08 & 0.05 & 0.473 \\
Composite unit cell & $0.858 \times 0.946 \times 0.875$ & \\
$\quad$ & & \\
\hline
\end{tabular}

horizontal shear mode, wave propagation is influenced by the components of along the $z$ direction, and the frequency is given by $\omega_{S H}=\frac{\sqrt{2}}{2} \sqrt{\frac{C_{66}+2 D_{311} k_{1}^{2}}{\rho^{*}}} k_{1}$.

These expressions of the phase velocities for the three modes of propagation reveal that the second order continuum is dispersive because the frequency is nonlinearly related to the wave number $\mathrm{k}$, due to the presence of second order elasticity constants. Observe that one recovers constant phase velocities for the first order Cauchy continuum (independent of the wave number) when only first order effects are considered.

We shall consider the following non-dimensional system parameters: $k L$ the dimensionless wave number. $\frac{\omega_{0} L}{\sqrt{\frac{E}{\rho}}}$ the dimensionless frequency, $\frac{c^{p}}{\sqrt{\frac{E}{\rho}}}, \frac{c^{g}}{\sqrt{\frac{E}{\rho}}}$ the dimensionless phase and group velocities respectively.E, $\rho, L$ Young modulus, density and length of the elementary cell respectively.

In the next section, 3D finite element models of textile based composites and 3D woven interlock are built to evaluate the first and second gradient of displacement stiffness tensors introduced in Eq. (2). The numerical homogenization technique consists in determining the overall effective first and second grade elastic coefficients over a representative unit cell (RUC) of microstructure materials with different sizes, relying on a finite element discretization of the unit cell geometry. Once the constitutive law is determined, the acoustics characteristics for each material can be presented based on Eq. (16).

Table 3

Predicted effective moduli $C_{i j}$ for the simulated 3D orthogonal composite unit cell.

\begin{tabular}{|c|c|c|c|c|c|c|c|c|c|}
\hline \multirow[t]{2}{*}{ Stiffness tensor $\mathrm{C}_{\mathrm{ij}}(\mathrm{MPa})$} & \multicolumn{3}{|c|}{ Uniaxial tensile tests } & \multicolumn{3}{|c|}{ Biaxial tensile tests } & \multicolumn{3}{|c|}{ Shear tests } \\
\hline & $\mathrm{C}_{11}$ & $\mathrm{C}_{22}$ & $\mathrm{C}_{33}$ & $C_{12}$ & $\mathrm{C}_{23}$ & $\mathrm{C}_{13}$ & $\mathrm{C}_{44}$ & $\mathrm{C}_{55}$ & $\mathrm{C}_{66}$ \\
\hline 3D orthogonal composite & 18,640 & 24,756 & 12,033 & 5101 & 5318 & 5428 & 2591 & 2506 & 2416 \\
\hline
\end{tabular}

Table 4

Equivalent second-order stiffness tensor components D of the 3D orthogonal composite. Units are in $\mathrm{N}$.

\begin{tabular}{|c|c|c|c|c|c|c|c|c|c|c|c|c|}
\hline$D_{111}$ & $\mathrm{D}_{222}$ & $\mathrm{D}_{333}$ & $\mathrm{D}_{122}$ & $\mathrm{D}_{133}$ & $D_{211}$ & $\mathrm{D}_{233}$ & $D_{311}$ & $\mathrm{D}_{322}$ & $\mathrm{D}_{112}$ & $\mathrm{D}_{113}$ & $\mathrm{D}_{123}$ & $\mathrm{D}_{212}$ \\
\hline 631 & 1003 & 622 & 403 & 205 & 306 & 244 & 274 & 360 & 1833 & 978 & 742 & 1999 \\
\hline $\mathrm{D}_{213}$ & $\mathrm{D}_{223}$ & $\mathrm{D}_{312}$ & $\mathrm{D}_{313}$ & $\mathrm{D}_{323}$ & & & & & & & & \\
\hline 725 & 1709 & 890 & 1462 & 1661 & & & & & & & & \\
\hline
\end{tabular}

Table 5

Predicted effective moduli $\mathrm{C}_{\mathrm{ij}}$ for the layered textile composite.

\begin{tabular}{|c|c|c|c|c|c|c|c|c|c|}
\hline \multirow[t]{2}{*}{ Stiffness tensor $\mathrm{C}_{\mathrm{ij}}(\mathrm{MPa})$} & \multicolumn{3}{|c|}{ Uniaxial tensile tests } & \multicolumn{3}{|c|}{ Biaxial tensile tests } & \multicolumn{3}{|c|}{ Shear tests } \\
\hline & $\mathrm{C}_{11}$ & $\mathrm{C}_{22}$ & $\mathrm{C}_{33}$ & $\mathrm{C}_{12}$ & $\mathrm{C}_{23}$ & $\mathrm{C}_{13}$ & $\mathrm{C}_{44}$ & $\mathrm{C}_{55}$ & $\mathrm{C}_{66}$ \\
\hline Layered textile composite & 36,652 & 36,977 & 12,597 & 8985 & 5844 & 5678 & 10,965 & 2463 & 2384 \\
\hline
\end{tabular}

Table 6

Equivalent second-order stiffness tensor components D of the simulated layered composite. Units in $\mathrm{N}$.

\begin{tabular}{|c|c|c|c|c|c|c|c|c|c|c|c|c|}
\hline $\mathrm{D}_{111}$ & $\mathrm{D}_{222}$ & $\mathrm{D}_{333}$ & $\mathrm{D}_{122}$ & $D_{133}$ & $\mathrm{D}_{211}$ & $D_{233}$ & $\mathrm{D}_{311}$ & $\mathrm{D}_{322}$ & $\mathrm{D}_{112}$ & $\mathrm{D}_{113}$ & $\mathrm{D}_{123}$ & $\mathrm{D}_{212}$ \\
\hline 41,095 & 41,325 & 49 & 15,766 & 32 & 15,847 & 31 & 5518 & 5275 & 64,506 & 6018 & 5656 & 65,111 \\
\hline $\mathrm{D}_{213}$ & $\mathrm{D}_{223}$ & $D_{312}$ & $\mathrm{D}_{313}$ & $D_{323}$ & & & & & & & & \\
\hline 5931 & 5774 & 11,057 & 20,384 & 19,990 & & & & & & & & \\
\hline
\end{tabular}



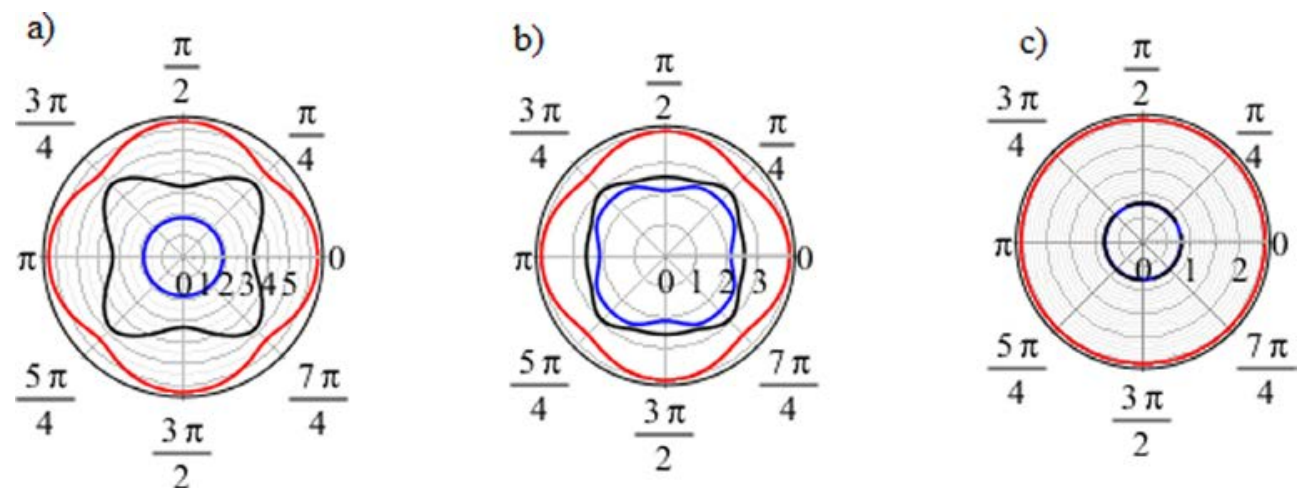

Fig. 5. Frequency plots for Layered textile composites versus angle $\theta$ for three value of $\chi$, a) $\chi=\pi / 2$, b) $\chi=\pi / 4$ and c) $\chi=0$.
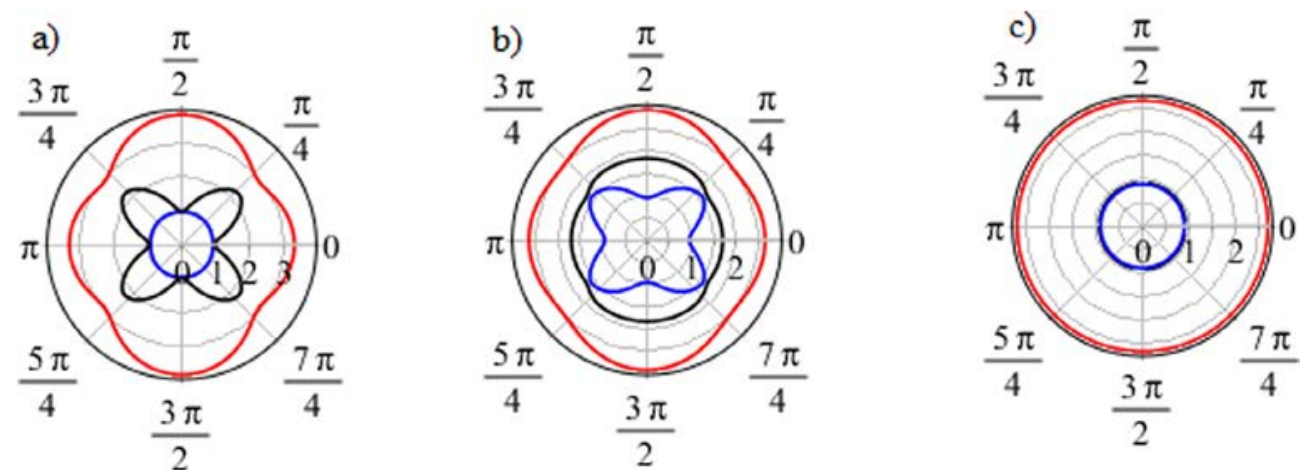

Fig. 6. Frequency band structure for $3 \mathrm{D}$ woven orthogonal interlock composite versus angle $\theta$ for three value of $\chi$, a) $\chi=\pi / 2$, b) $\chi=\pi / 4$ and c) $\chi=0$.

Table 7

Phase and group velocity for a direction of wave propagation perpendicular to $\left(\mathrm{k}_{1}, \mathrm{k}_{2}\right)$ plane for the three modes.

\begin{tabular}{lll}
\hline & Phase velocity & Group velocity \\
\hline Longitudinal mode & $C_{l}^{p}=\sqrt{\frac{C_{33}+D_{333} k_{3}^{2}}{\rho}}$ & $C_{l}^{g}=\frac{2 k_{3} C_{33}+4 k_{3}^{3} D_{333}}{2 \rho \sqrt{\frac{k_{3}^{2} C_{33}+k_{3}^{4} D_{333}}{\rho}}}$ \\
Vertical shear mode & $C_{S V}^{p}=\sqrt{\frac{\frac{C_{55}}{2}+D_{233} k_{3}^{2}}{\rho}}$ & $C_{S V}^{g}=\frac{k_{3} C_{55}+4 k_{3}^{3} D_{233}}{2 \rho \sqrt{\frac{k_{3}^{2} \frac{C_{55}}{2}+k_{3}^{4} D_{233}}{\rho}}}$ \\
Horizontal shear mode & $C_{S H}^{p}=\sqrt{\frac{\frac{C_{66}}{2}+D_{133} k_{3}^{2}}{\rho}}$ & $C_{S H}^{g}=\frac{k_{3} C_{66}+4 k_{3}^{3} D_{133}}{2 \rho \sqrt{\frac{k_{3}^{2} \frac{C_{66}}{2}+k_{3}^{4} D_{133}}{\rho}}}$ \\
\hline
\end{tabular}

\section{Applications}

The analysis of dispersive effects in microstructured solids like composites can be performed for a wide class of composites, based on the identification of the second order gradient effective properties. In order to illustrate the proposed methodology, we shall next focus on the wave propagation characteristics of 3D orthogonal interlock composites and layered textile composites (see Figs. 3 and 4).
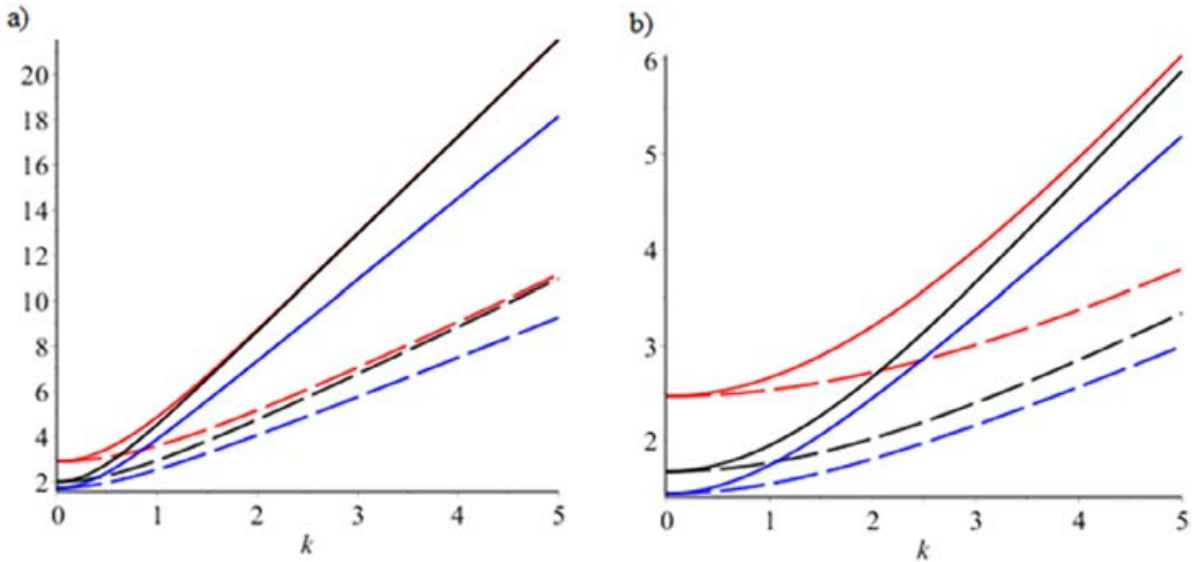

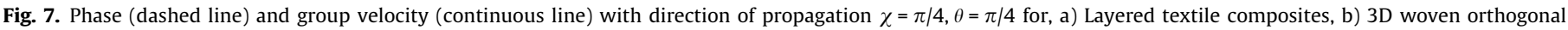
interlock composites, Red line: longitudinal mode, black line: vertical shear mode, blue line: horizontal shear mode. 


\subsection{D woven orthogonal interlock composite}

The first- and second-order elasticity tensors C and D of 3D woven orthogonal interlock are derived computationally using the FE method. The corresponding input material properties for yarns (E-glass) and matrix (epoxy) are given in Table 1.

The Yarn cross sections are considered constant and uniform along the yarn path and perfect bonding is assumed between the yarns and matrix. The dimensions of the geometric unit cell model are listed in Table 2 ,
The values of the computed classical moduli $\mathrm{C}_{\mathrm{ij}}$ are given for the 3D composite in Table 3.

The values of the computed second order moduli $D_{i j}$ for the $3 D$ composite are given in Table 4.

\subsection{Layered textile composites}

In this application, we combine two textiles into one. We create the first layer of a twill weave with yarn spacing of $1 \mathrm{~mm}$, width of $0.8 \mathrm{~mm}$ and thickness of $0.1 \mathrm{~mm}$. We next create the weave
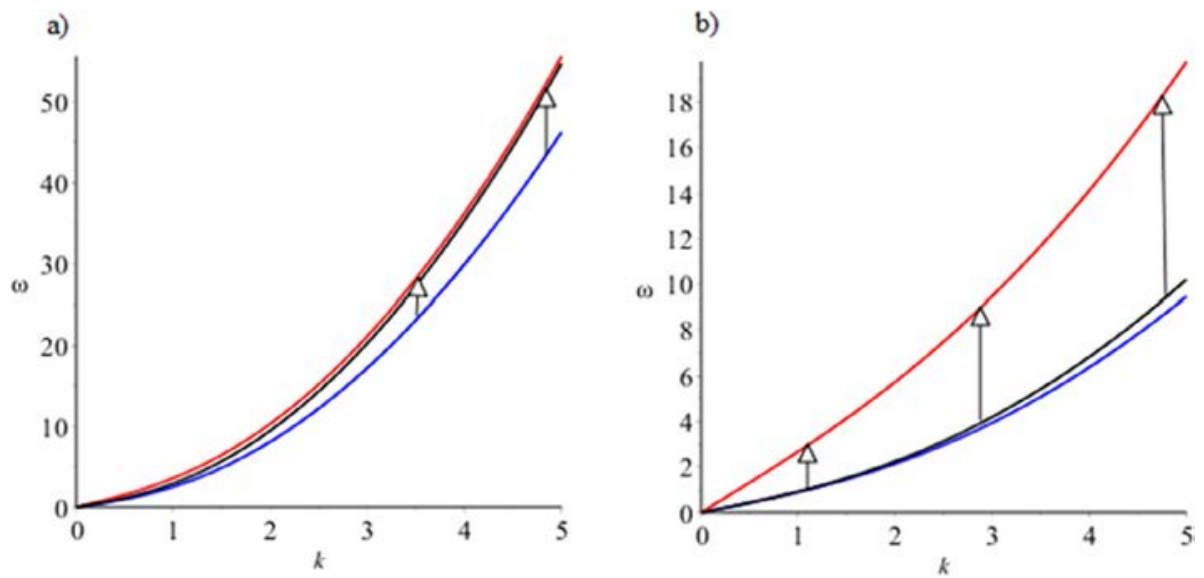

Fig. 8. Frequency band structure with direction of propagation $\chi=\pi / 4, \theta=\pi / 4$ for a) Layered textile composites, b) 3D woven orthogonal interlock composites.

\section{Layered textile composites}
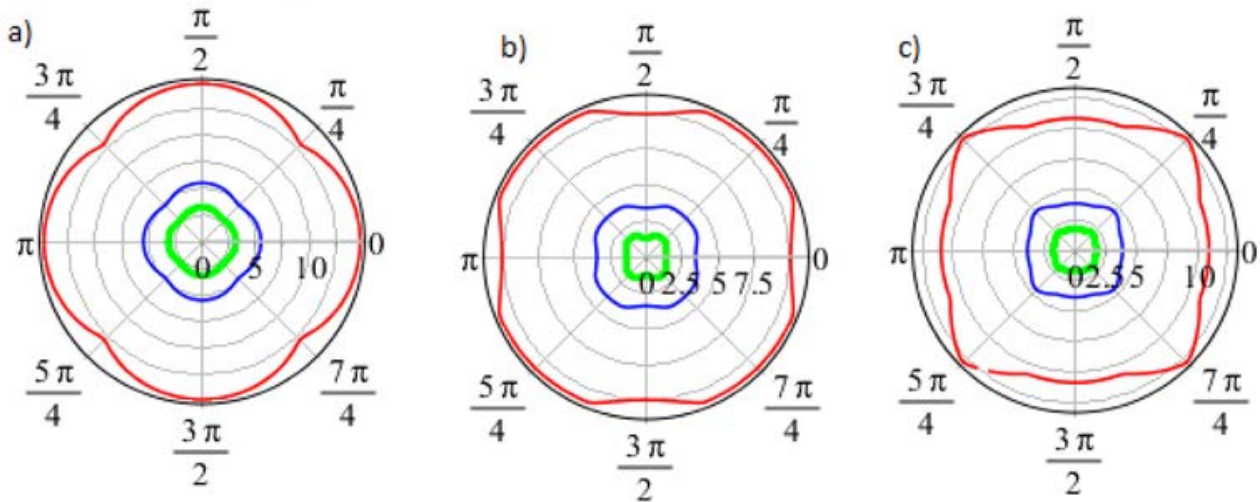

\section{D woven orthogonal interlock composite}
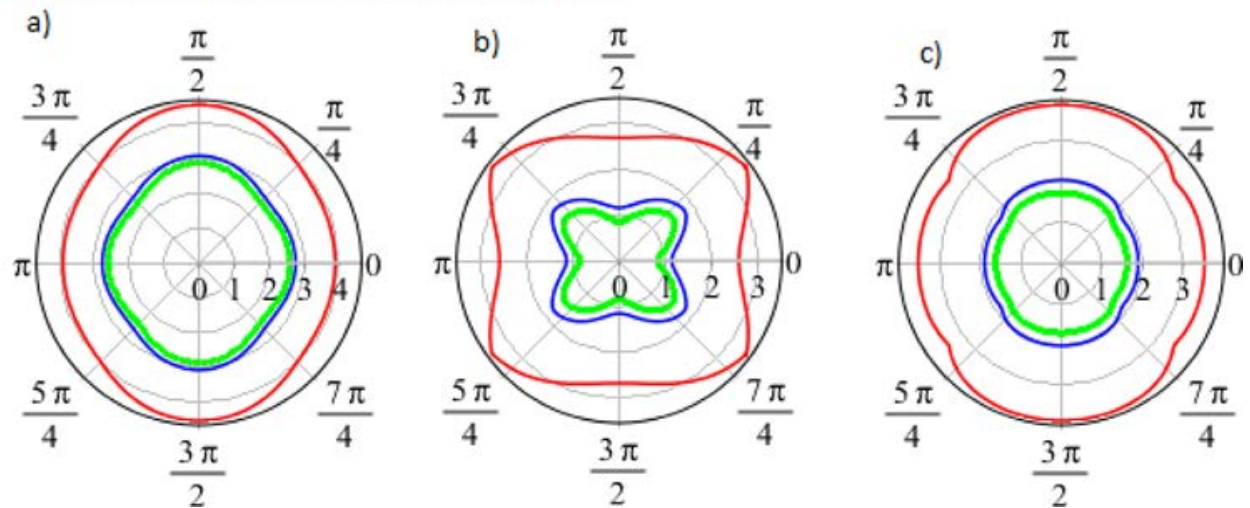

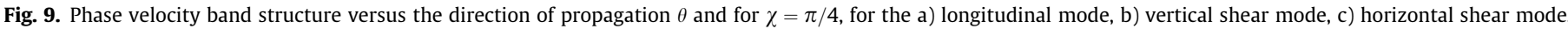
Green line: Cauchy medium (for all values of $\mathrm{k}$ ). Blue line: second gradient medium with wavenumber $\mathrm{k}=1$. Red line: second gradient medium with wave number $\mathrm{k}=3$. 


\section{Layered textile composites}

-Second gradient medium
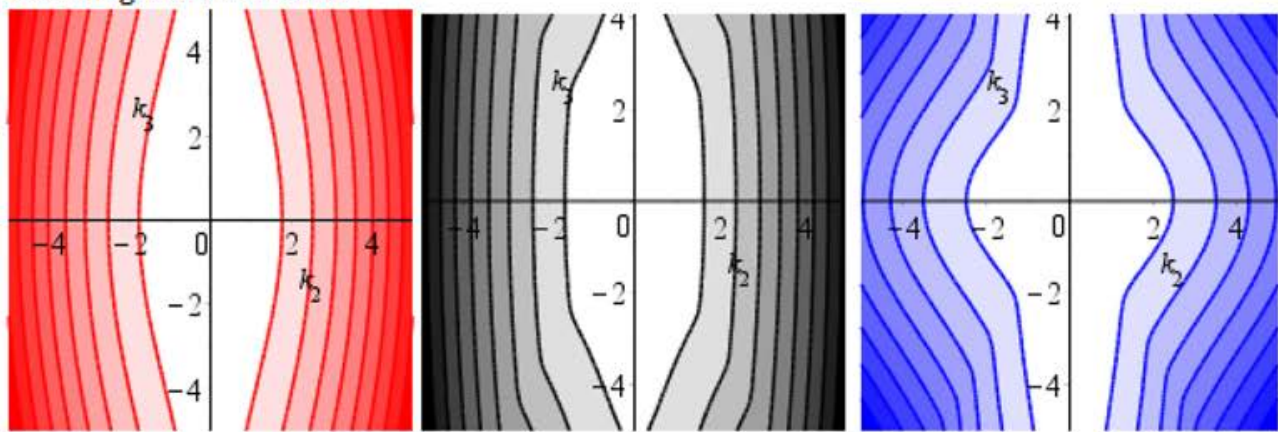

-Cauchy medium
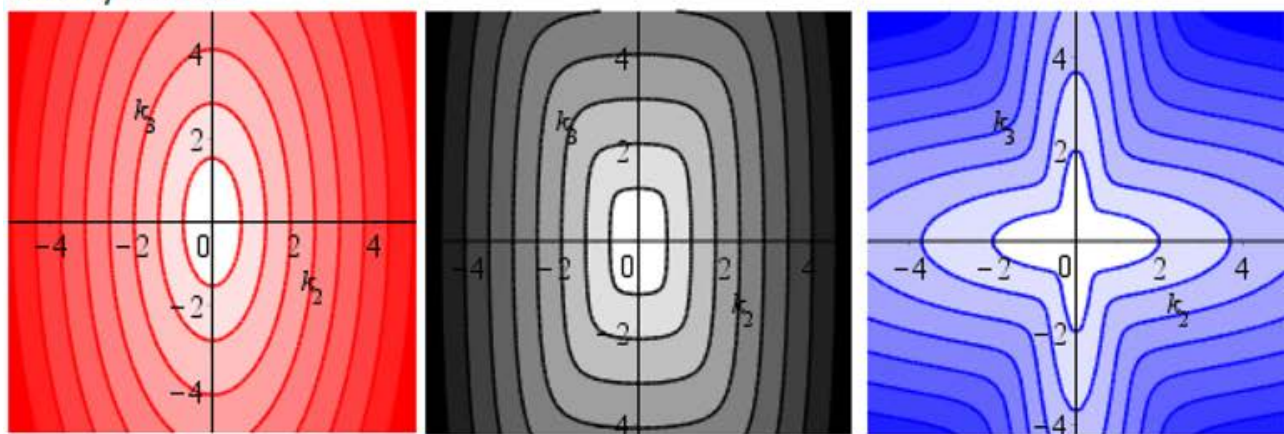

\section{D w oven orthogonal interlock composite}

\section{-Second gradient medium}
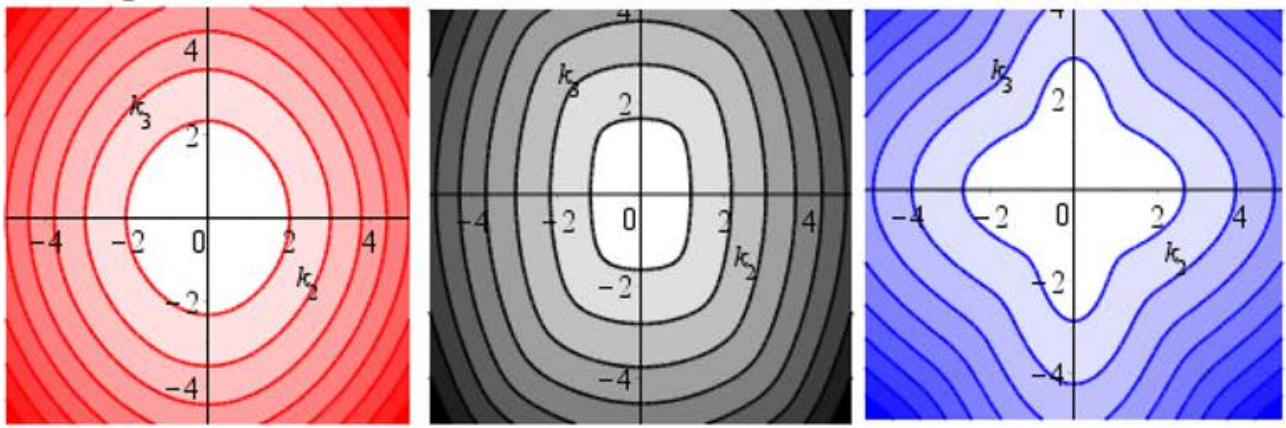

-Cauchy medium
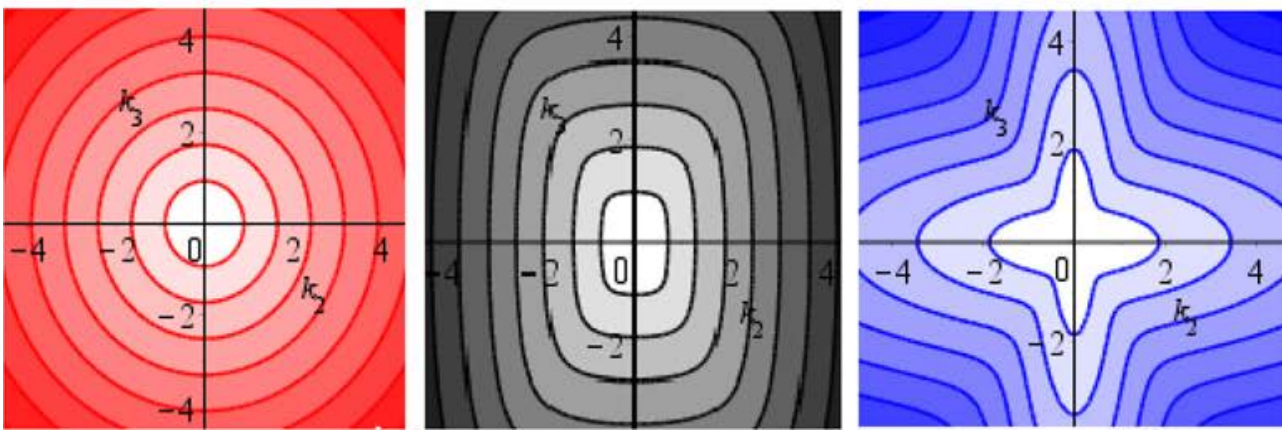

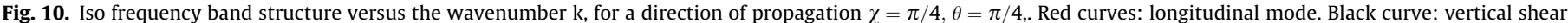
mode. Blue curve: horizontal shear mode. (For interpretation of the references to colour in this figure caption, the reader is referred to the web version of this article.)

pattern for the second layer, in this case a plain weave is considered, taken the same yarn spacing and dimensions as twill. The produced textile is then impregnated with a resin matrix (see Fig. 4).
The computed values of classical moduli $\mathrm{C}_{\mathrm{ij}}$ and of the secondorder elasticity tensor coefficients are given in Table 5 and Table 6 successively. 


\subsection{Dispersion relation for 3D orthogonal and layered textile composites}

The frequency band structure, the group and phase velocities can be obtained for the two investigated structure based on the previous methodology after computing the moduli of the homogenized second gradient continuum. In the sequel, the red line corresponds to the longitudinal mode, the black line for the vertical shear and the blue line to the horizontal shear mode.

For in-plane propagation with $\chi=\pi / 2$ (in the $\left(\mathrm{k}_{1}, \mathrm{k}_{2}\right)$ plane), the longitudinal and vertical shear modes are coupled to each other via coefficient $\mathrm{C}_{12}$ (it is Poisson ratio in $\left(\mathrm{k}_{1}, \mathrm{k}_{2}\right)$ plane) and the shear coefficient $\mathrm{C}_{44}$ in the same plane, which entails the irregular shape in the frequency diagram and explains the anisotropic behavior of the investigated structures for these two modes. In contrast, the horizontal shear is directly proportional to the shear coefficient $\mathrm{C}_{55}$ and $\mathrm{C}_{66}$ in the $\left(\mathrm{k}_{2}, \mathrm{k}_{3}\right)$ plane and $\left(\mathrm{k}_{1}, \mathrm{k}_{3}\right)$ plane respectively, so that the two structures behaves isotropically, as witnessed by the circular shape of polar plot of the phase velocity in Figs. 5 and 6. The second order rigidity matrix does not affect the coupling between modes and leads to a hyperbolic relation between the frequency and wave number for each mode. For a propagation in 3D space, the three modes (longitudinal, vertical and horizontal shear) are coupled to each to other via the coefficients $C_{12}, C_{13}, C_{23}$ and the shear coefficient $\mathrm{C}_{44}, \mathrm{C}_{55}, \mathrm{C}_{66}$, which explains the appearance of the irregular shape in the 3 modes (anisotropic behavior in the three modes). For $\chi=0$ (case of propagation perpendicular to the $\left(k_{1}\right.$, $\mathrm{k}_{2}$ ); the longitudinal mode is influenced by the first and second order tensile rigidity in the $\mathrm{z}$ direction, with frequency
$\omega_{l}=\sqrt{\frac{C_{33} k_{3}^{2}+D_{333} k_{3}^{4}}{\rho}}$; the vertical shear mode is influenced by the first and second order shear coefficients, with frequency $\omega_{S V}=\sqrt{\frac{\frac{C_{55}}{2} k_{3}^{2}+D_{233} k_{3}^{4}}{\rho}}$ and similar effects also occur for the horizontal shear mode, with the corresponding frequency given versus the first and second order coefficients $\omega_{S H}=\sqrt{\frac{\frac{c_{66}}{2} k_{3}^{2}+D_{133} k_{3}^{4}}{\rho}}$. These expressions of the frequencies for the three modes reveal the isotropic behavior of the two considered structures for this direction of propagation; the second order rigidity properties do not play any role in the coupling between modes.

A relation between the phase and group velocities is obtained after a lengthy calculation starting from the dynamical equilibrium equation and the definition of the group velocity.

We give in Table 7 the expression of the phase and group velocity in each mode versus the first and second order rigidity constants for a direction of propagation perpendicular to the $\left(\mathrm{k}_{1}, \mathrm{k}_{2}\right)$ plane $(\chi=0)$.

The difference between the phase and group velocities is clear from Table 7, evidencing the dispersive behavior of the second order gradient medium.

We next demonstrate the dispersive behavior of the second order gradient medium in the general situation of wave propagation in 3D space, by plotting the phase and group velocity versus the wave number for the two investigated structures.

It can be observed from Fig. 7 that the group velocity differs from the phase velocity for the layered textile composites and the 3D woven orthogonal interlock composites, for each modes

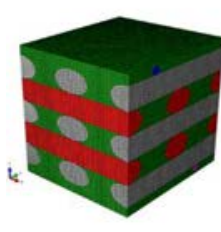

(a)

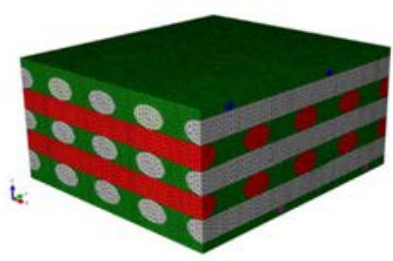

(b)

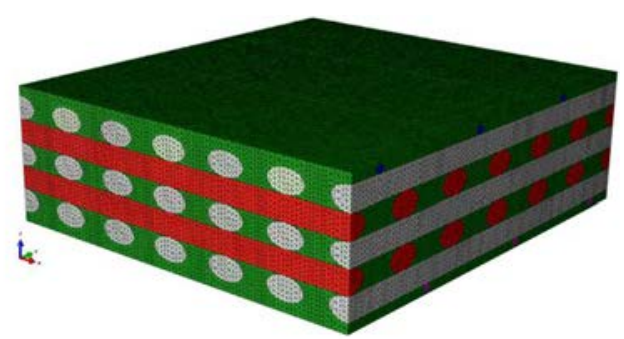

(c)

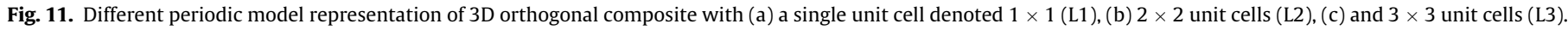

Table 8

Calculated equivalent moduli $\mathrm{C}_{\mathrm{ij}}(\mathrm{MPa})$ for 3D orthogonal interlock composites with different sample sizes.

\begin{tabular}{|c|c|c|c|c|c|c|c|c|c|}
\hline \multirow[t]{2}{*}{ RUCs sizes } & \multicolumn{3}{|c|}{ Uniaxial tensile tests } & \multicolumn{3}{|c|}{ Biaxial tensile tests } & \multicolumn{3}{|c|}{ Shear tests } \\
\hline & $\mathrm{C}_{11}$ & $\mathrm{C}_{22}$ & $\mathrm{C}_{33}$ & $\mathrm{C}_{12}$ & $\mathrm{C}_{23}$ & $\mathrm{C}_{13}$ & $\mathrm{C}_{44}$ & $\mathrm{C}_{55}$ & $\mathrm{C}_{66}$ \\
\hline $\mathrm{L} 1(1 \times 1)$ & 18,640 & 24,756 & 12,033 & 5101 & 5318 & 5428 & 2591 & 2506 & 2416 \\
\hline $\mathrm{L} 2(2 \times 2)$ & 18,378 & 24,242 & 11,893 & 4899 & 5150 & 5274 & 2585 & 2500 & 2417 \\
\hline L3 $(3 \times 3)$ & 18,292 & 24,080 & 11,847 & 4834 & 5096 & 5223 & 2584 & 2498 & 2418 \\
\hline
\end{tabular}

Table 9

Equivalent second-order stiffness tensor components D of the simulated 3D orthogonal composite unit cells. Units in N.

\begin{tabular}{|c|c|c|c|c|c|c|c|c|c|}
\hline RUCs sizes & $\mathrm{D}_{111}$ & $\mathrm{D}_{222}$ & $D_{333}$ & $\mathrm{D}_{122}$ & $\mathrm{D}_{133}$ & $\mathrm{D}_{211}$ & $\mathrm{D}_{233}$ & $\mathrm{D}_{311}$ & $D_{322}$ \\
\hline $1 \times 1$ & 631 & 1003 & 622 & 403 & 205 & 306 & 244 & 274 & 360 \\
\hline $2 \times 2$ & 2361 & 3798 & 345 & 1158 & 149 & 878 & 168 & 893 & 1150 \\
\hline $3 \times 3$ & 6143 & 9905 & 237 & 2310 & 118 & 1778 & 130 & 1845 & 2345 \\
\hline RUCs sizes & $D_{112}$ & $\mathrm{D}_{113}$ & $\mathrm{D}_{123}$ & $\mathrm{D}_{212}$ & $D_{213}$ & $D_{223}$ & $D_{312}$ & $\mathrm{D}_{313}$ & $D_{323}$ \\
\hline $1 \times 1$ & 1833 & 978 & 742 & 1999 & 725 & 1709 & 890 & 1462 & 1661 \\
\hline $2 \times 2$ & 6509 & 1454 & 1581 & 7036 & 1468 & 2362 & 2544 & 4013 & 4708 \\
\hline $3 \times 3$ & 14,246 & 2355 & 2830 & 15,427 & 2558 & 3510 & 4899 & 8043 & 9568 \\
\hline
\end{tabular}


(a)

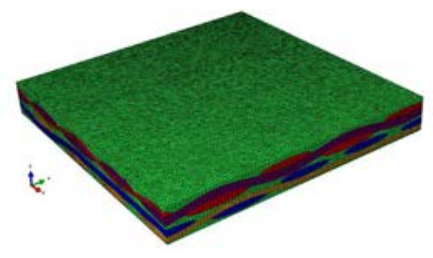

(b)
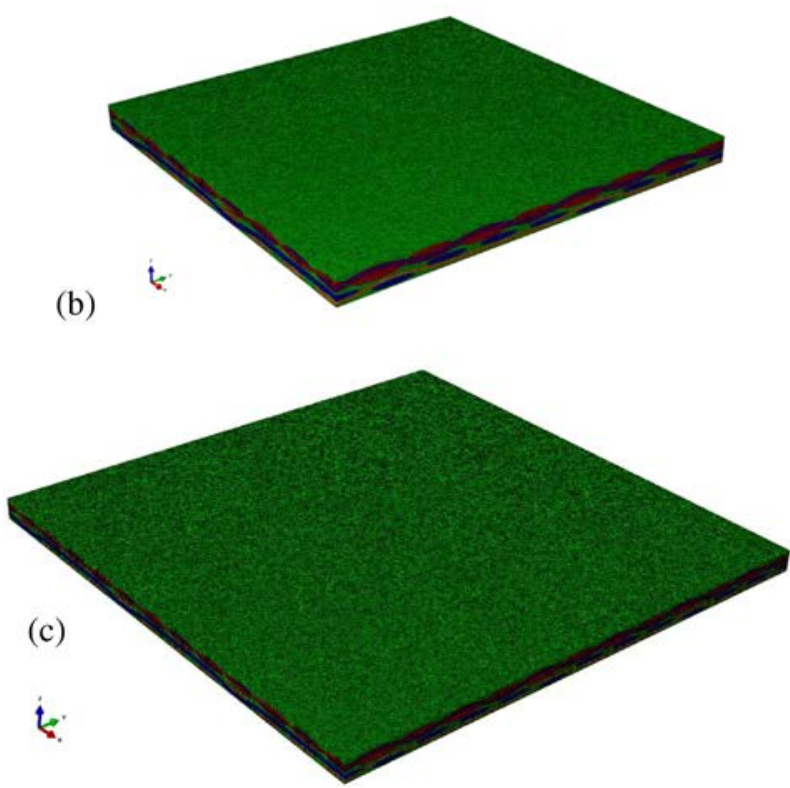

Fig. 12. Different periodic model representation of layered composite with (a) a single unit cell $1 \times 1$, (b) $2 \times 2$ unit cells, (c) and $3 \times 3$ unit cells.

of propagation; this entails the dispersive behavior of the second order medium.

The band structure evaluated for the two investigated structures is shown in Fig. 8 with a direction of propagation $\chi=\pi / 4, \theta=\pi / 4$. A non-complete band gap occurs between the different branches; the excess in partial band gap exhibited by 3D woven orthogonal interlock composite due to the presence of large area between the longitudinal and vertical shear modes.

These results clearly have a validity limited to the low frequencies range, to ensure the validity of the obtained homogenized model.

\section{Comparison between second gradient medium and Cauchy medium}

We compare in Fig. 9 the phase velocity for the three modes of propagation for the Cauchy and second gradient substitution continua for the two investigated composites, in order to investigate the effect of the second gradient terms on the dispersive behavior of propagating waves.

We observe an important shift in the phase velocity between Cauchy effective medium and the second order medium, since the phase velocity is increased by the second order effective modulus. The shape change of the phase velocity when increasing the wave number from $\mathrm{k}=1$ to $\mathrm{k}=3$ shows the dispersive behavior of the second gradient medium, while Cauchy medium is non dispersive.

In a second step, we investigate the effect of the second gradient on the anisotropic behavior of the material from the plot of the isofrequency contour for the two investigated composites in the case of second gradient and Cauchy effective medium (Fig. 10).

We observe in Fig. 10 that irregular shapes of the iso-frequency plots are obtained for both effective continua and for the three modes of propagation, so that the anisotropic behavior of the structures does not depend on the incorporation of second order terms.

\section{Effect of the RUC size on the dynamical behavior}

We next investigate the effect of the RUC size on wave propagation properties, by considering RUCs including an increasing number of repeat of the unit cells of the 3D orthogonal composite and 3D textile pattern.

\subsection{D through-thickness orthogonal woven composite}

First and second gradient elastic moduli are evaluated for different sizes of through-thickness orthogonal interlock composites, obtained by repeating the composite elementary pattern in the plane orthogonal to the direction of pile up (Fig. 11).

The first order effective moduli are shown to size independent (Table 8), whereas the second order moduli show a strong sizedependency, since they are all increasing with the RUC size without showing any tendency to stabilize (consideration of the effect of larger RUC sizes involves a huge computational cost, and will not be investigated in this contribution).

Table 10

Calculated equivalent moduli $\mathrm{C}_{\mathrm{ij}}(\mathrm{MPa})$ for layered composites with different sample sizes.

\begin{tabular}{|c|c|c|c|c|c|c|c|c|c|}
\hline \multirow[t]{2}{*}{ RUCs sizes } & \multicolumn{3}{|c|}{ Uniaxial tensile tests } & \multicolumn{3}{|c|}{ Biaxial tensile tests } & \multicolumn{3}{|c|}{ Shear tests } \\
\hline & $\mathrm{C}_{11}$ & $\mathrm{C}_{22}$ & $C_{33}$ & $\mathrm{C}_{12}$ & $\mathrm{C}_{23}$ & $\mathrm{C}_{13}$ & $\mathrm{C}_{44}$ & $\mathrm{C}_{55}$ & $\mathrm{C}_{66}$ \\
\hline $1 \times 1$ & 36,652 & 36,977 & 12,597 & 8985 & 5844 & 5678 & 10,965 & 2463 & 2384 \\
\hline $2 \times 2$ & 36,596 & 36,920 & 12,596 & 8971 & 5853 & 5686 & 10,978 & 2468 & 2389 \\
\hline $3 \times 3$ & 36,578 & 36,901 & 12,596 & 8966 & 5857 & 5689 & 7831 & 2470 & 2391 \\
\hline
\end{tabular}

Table 11

Equivalent second-order stiffness tensor components D of the simulated layered composite unit cells. Units in N.

\begin{tabular}{|c|c|c|c|c|c|c|c|c|c|}
\hline RUCs sizes & $\mathrm{D}_{111}$ & $\mathrm{D}_{222}$ & $\mathrm{D}_{333}$ & $\mathrm{D}_{122}$ & $\mathrm{D}_{133}$ & $\mathrm{D}_{211}$ & $\mathrm{D}_{233}$ & $\mathrm{D}_{311}$ & $\mathrm{D}_{322}$ \\
\hline $1 \times 1$ & 41,095 & 41,325 & 49 & 15,766 & 32 & 15,847 & 31 & 5518 & 5275 \\
\hline $2 \times 2$ & 187,352 & 187,017 & 25 & 63,513 & 17 & 63,533 & 17 & 17,928 & 17,514 \\
\hline $3 \times 3$ & 435,149 & 433,852 & 17 & 142,627 & 12 & 142,591 & 11 & 37,016 & 36,512 \\
\hline RUCs sizes & $\mathrm{D}_{112}$ & $\mathrm{D}_{113}$ & $\mathrm{D}_{123}$ & $\mathrm{D}_{212}$ & $\mathrm{D}_{213}$ & $\mathrm{D}_{223}$ & $\mathrm{D}_{312}$ & $D_{313}$ & $D_{323}$ \\
\hline $1 \times 1$ & 64,506 & 6018 & 5656 & 65,111 & 5931 & 5774 & 11,057 & 20,384 & 19,990 \\
\hline $2 \times 2$ & 261,873 & 18,415 & 17,890 & 261,822 & 18,427 & 18,004 & 35,794 & 74,547 & 74,103 \\
\hline $3 \times 3$ & 590,250 & 37,499 & 36,794 & 589,254 & 37,685 & 36,999 & 73,960 & 162,624 & 162,128 \\
\hline
\end{tabular}




\subsection{Layered textile composite}

The same analysis of size effects is repeated for layered composites, whereby the elementary pattern is repeated in the main plane of the composite to generate three RUC's with increasing sizes (Fig. 12).

The same conclusions can here be repeated: the first order moduli show a size independent behavior (Table 10), whereas a strong increase with size of the second order moduli is obtained (Table 11).

In order next to evidence size effects on the dynamical behavior (dispersion and anisotropic behavior), the frequency band structure is determined for three RUC sizes versus the direction of propagation $\theta$ (for $\chi=\frac{\pi}{4}$ ), for two values of wave number k (in Fig. 13 for the 3D interlock and in Fig. 14 for the layered textile composite). The observed change in frequency in (Figs. 13 and 14) with RUC size changes is due to the significant change of the second order rigidity matrix (Table 9, Table 11), since the different structures are selected to have the same effective density.

In order to analyze the effect of the RUC size on the band gap, we plot in the Fig. 15 the frequency band structure for two different sizes. Inspection of Fig. 15a,b shows that the RUC size does not influence the partial band gap between different modes; the area between the three modes in L1 is indeed approximately the same as the band gap between the three modes in L3. For the Cauchy medium, the dispersion relation is size-independent, since the effective rigidity matrix in Tables 8 and 10 does not vary with size. We are thus entitled to conclude that the dispersive behavior of the structure only depends on the nature of the chosen effective medium. To the contrary, the anisotropic behavior of the effective medium is not impacted by the RUC size.

\section{Conclusion}

We analyse in the present contribution the characteristics of waves propagating within 3D textiles composites in the low frequency range, based on identified second order anisotropic effective media. The obtained band diagram show that the microstructure give rise to dispersion effects which would not be captured by Cauchy continua. The effective static properties of the studied textile composites are obtained computationnally by an equivalent strain energy method, relying on [16]. Plots of the iso-frequency contour for the two investigated composites in the case of second gradient and Cauchy effective medium show that the second gradient contributions does not modifiy the anisotropic behavior of the considered composites. Important size effects on the dynamical behavior are shown, especially reflected by the dispersive behavior and the anisotropic dynamic responses, due to the significant overall increase of the second order rigidity matrix when increasing the RUC size.

The consideration of a viscous matrix leading to an effective continuum showing long range memory effects deserves future contributions.
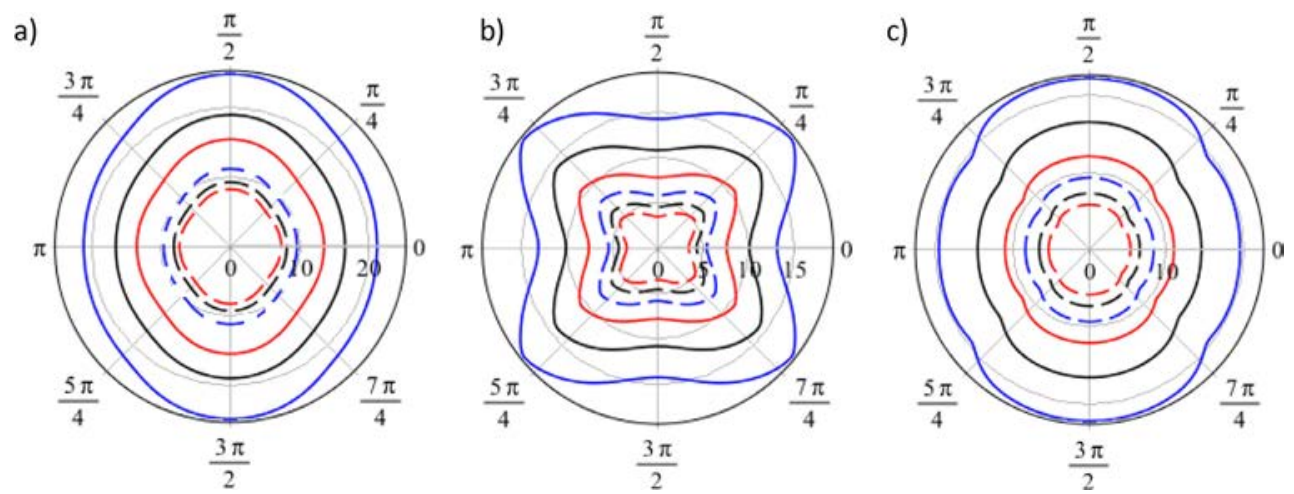

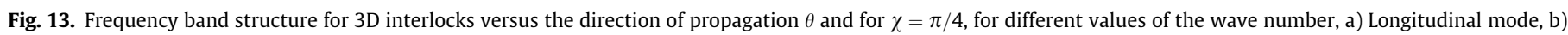
Vertical shear mode, c) Horizontal shear mode. Continuous line: $\mathrm{k}=4$, Dashed line: $\mathrm{k}=2$. Red line: L1, Black line: L2, Blue line: L3.
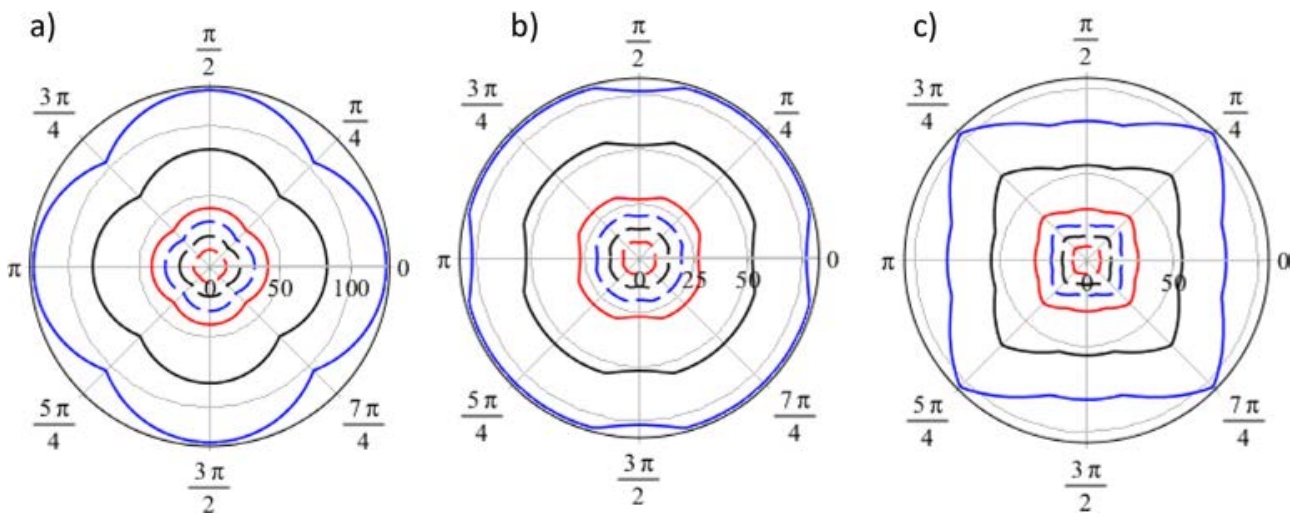

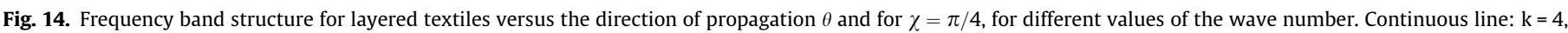
Dashed line: $\mathrm{k}=2$. a) Longitudinal mode, b) Vertical shear mode, c) Horizontal shear mode. Red line: L1, Black line: L2, Blue line: L3. 


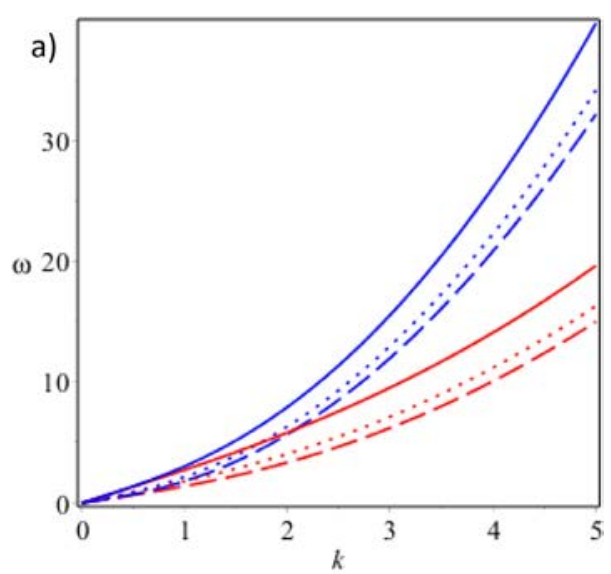

b)

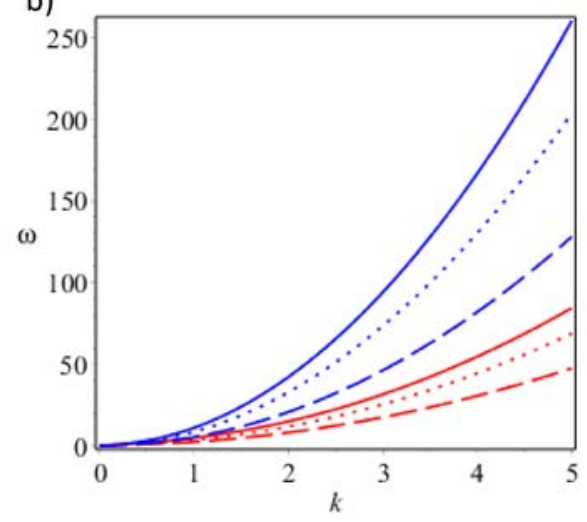

Fig. 15. Frequency band structure in function of wave number $\mathrm{k}$ for $\chi=\pi / 3, \theta=\pi / 6$, a) 3D interlock, b) layered textile. Red line: L1, Blue line: L3. Continuous line: longitudinal mode, dotted line: vertical shear mode. Dashed line: horizontal shear mode. (For interpretation of the references to colour in this figure legend, the reader is referred to the web version of this article.)

\section{References}

[1] Vasseur JO, Deymier PA, Chenni B, Djafar-Rouhani B, Dobrwnski L, Prevost D. Experimental and theoretical evidence for the existence of absolute acoustic band gaps in two-dimensional solid phononic crystals. Phys Rev Lett 2011;86:3012-5.

[2] Maldovan M. Sound and heart revolution in phononics 2013; 503: 209-17.

[3] Mei J, Ma G, Yang M, Yang Z, Wen W, Sheng P. Dark acoustic metamaterials as super absorbers for low-frequency sound. Nat Commun 2012;3:7.

[4] Clayes C, Deckers E, Pluymers B, Desmet W. A lightweight vibro-acoustic metamaterial demonstrator: Numerical and experimental investigation. Mech Syst Signal Process 2016;70-71:853-80.

[5] Sigalas MM, Economu EN. Elastic and acoustic wave band structure. J Sound Vib 1992;158:377-82.

[6] Liu ZY, Zhang XX, Mao YW, Zhu YY, Yang ZY, Chang CT, et al. Locally Resonant Sonic Mater Sci 2000;289:1734-6.

[7] Hussein MI, Leamy MJ, Ruzzene M. Dynamic of phononic materials and structures: historical origins, recent progress, and future outlook. Appl Mech Rev 2014;66:1-38.

[8] Mace BR, Duhamel D, Brennan MJ, Hinke L. Finite element prediction of wave motion in structural waveguides. J Acoust Soc Am 2005;117:2835-42.
[9] Lee SK, Mace BR, Brennan MJ. Wave propagation, reflection and transmission in non-uniform one-dimensional waveguides. J Sound Vib 2007:304:31-49.

[10] Mencik JM, Ichchou MN. A substructuring technique for finite element wave propagation in multi-layered systems. Comput Methods Appl Mech Eng 2008;197:502-23.

[11] Mencik JM, Duhamel D. A wave-based model reduction technique for the description of the dynamic behavior of periodic structures involving arbitraryshaped substructures and large-sized finite element models. Finite Elem Anal Des 2015:101:1-14.

[12] Silva PB, Mencik JM, Arruda JRF. Wave finite element-based super elements for forced response analysis of coupled systems via dynamic substructuring. Int J Numer Methods Eng. John Wiley Sons Ltd; 2015.

[13] Pasternak E, Mühlhaus HB. Generalised homogenisation procedures for granular materials. J Eng Math 2005;52:199-229.

[14] Pasternak E, Mühlhaus HB, Dyskin AV. Phil Mag 2006;86(21):3339-71.

[15] Reda H, Rahali Y, Ganghoffer JF, Lakiss H. Analysis of dispersive waves in repetitive lattices based on homogenized second- gradient continuum models. Compos Struct 2016;152:712-28.

[16] Goda I, Ganghoffer JF Construction of first and second order grade anisotropic continuum media for 3D porous and textile composite structures. Compos Struct 2016;141:292-327.

[17] Metrikine AV, Askes H. One-dimensional dynamically consistent gradient elasticity models derived from a discrete microstructure: Part 1: generic formulation. Eur J Mech - A/Solids 2002;24(4):555-72.

[18] Metrikine AV. On causality of the gradient elasticity models. J Sound Vib 2006:297:727-42.

[19] Ostoja-Starzewki M. Lattice models in micromechanics. Appl Mech Rev 2002;55(1):35-60.

[20] Papargyri-Beskou S, Polyzos D, Beskos DE. Wave dispersion in gradient elastic solids and structures: a unified treatment. Int J Sol Struct 2009;46:3751-9.

[21] Andrianov I, Awrejcewicz J, Weichert D. Improved continuous models for discrete media. Math Prob Eng 2010;986242.

[22] Askes H, Aifantis EC. Gradient elasticity in statics and dynamics: an overview of formulations, length scale identification procedures, finite element implementations and new results. Int J Sol Struct 2011;48:1962-90.

[23] Bacigalupo A, Gambarotta L. Homogenization of periodic hexa-and tetrachiral cellular solids. Compos Struct 2014:116:461-76.

[24] Bacigalupo A, Bellis M. Auxetic anti-tetrachiral cellular solids: equivalent elastic properties and frequency band-gaps. Compos Struct 2015;131:530-44.

[25] Gutkin M, Aifantis E. Dislocations in the theory of gradient elasticity. Scr Mater 1999;40:559-66.

[26] Gourgiotis P, Georgiadis H. Plane-strain crack problems in microstructured solids governed by dipolar gradient elasticity. J Mech Phys Solids 2009:57:1898-920.

[27] Gitman I, Askes H, Aifantis E. Gradient elasticity with internal length and internal inertia based on the homogenisation of a representative volume element. J Mech Behav Mater 2007;18:1-16.

[28] Fafalis D, Filopoulos S, Tsamasphyros G. On the capability of generalized continuum theories to capture dispersion characteristics at the atomic scale. Eur J Mech - A/Solids 2012;36:25-37.

[29] Brillouin L. Wave propagation in periodic structures. New York: Dover; 1946.

[30] Chen W, Fish J. A dispersive model for wave propagation in periodic heterogeneous media based on homogenization with multiple spatial and temporal scales. J Appl Mech 2001;68:153-61.

[31] Fish J, Chen W, Nagai G. Non-local dispersive model for wave propagation in heterogeneous media: one-dimensional case. Int J Numer Methods Eng 2002;54:331-46.

[32] Gonella S, Ruzzene M. Multicell homogenization of one-dimensional periodic structures. J Vib Acoust 2010;132:011003.

[33] Gonella S, Greene M, Liu W. Characterization of heterogeneous solids via wave methods in computational microelasticity. J Mech Phys Solids 2011:59:959-74.

[34] Reda H, Rahali Y, Ganghoffer JF, Lakiss H. Wave propagation in 3D viscoelastic auxetic and textile materials by homogenized continuum micropolar models. Compos Struct 2016;141:328-45.

[35] Graff KF. Wave motion in elastic solids. London: Oxford University Press; 1975.

[36] Erofeyev VI. Wave processes in solids with microstructure. Singapore: World Scientific; 2003.

[37] Jakata K, Every AG. Determination of the dispersive elastic constants of the cubic crystals Ge, Si, GaAs, and In Sb. Phys Rev B 2008;77:174301. 